\title{
Bare Finger 3-Dimentional Touch with Multi-wavelength Sensing
}

\author{
Guo-Zhen Wang ${ }^{1}$, Hsuan-He Fang ${ }^{2}$, Yi-Pai Huang ${ }^{3}$, Kuo-Jui Hu ${ }^{4}$, Tsu-Han Chen ${ }^{5}$ \\ ${ }^{1}$ Institute of Electronics Engineering, National Chiao Tung University, Hsinchu 30010, Taiwan, R.O.C. \\ ${ }^{2}$ Institute of Electro-Optical Engineering, National Chiao Tung University, Hsinchu 30010, Taiwan, R.O.C. \\ ${ }^{3}$ Display Institute, National Chiao Tung University, Hsinchu 30010, Taiwan, R.O.C. \\ ${ }^{4}$ Color Lab, Industrial Technology Research Institute, Hsinchu 30010, Taiwan, R.O.C. \\ ${ }^{5}$ School of Electrical and Computer Engineering, Cornell University, Ithaca, NY 14853, USA
}

\begin{abstract}
Recent $3 D$ touch systems could only be applied to large display, none of those is suitable for portable devices. Therefore, we presented a $3 D(x, y, z)$ near distance touch system for portable devices. In the proposed system, multi-wavelength sensing was used to reduce capturing time of bare finger $2 D / 3 D$ touch system. The resolution in xy-plane and along z-axis is $2 \mathrm{~mm}$ and $4 \mathrm{~mm}$ individually. Moreover, a 4 inch 3D LCD with the 3-dimentional finger touch system was successfully demonstrated.
\end{abstract}

\section{Author Keywords}

3D touch; optical sensor; reflecting mode; bare finger; color filter; multi-wavelength; fast response.

\section{Introduction}

Touch system is the interface between users and machines. In recent years, touch system is common in devices such as TV games, mobile devices, medical instruments, etc. Because of the intuitive user interface provided by touch system, interaction could be performed more naturally. However, touchscreens nowadays could only detect $2 \mathrm{D}$ information $(\mathrm{x}, \mathrm{y})$, which is insufficient for 3D display where images emerge from the panel. Therefore, to interact with 3D images, 3D touch $(\mathrm{x}, \mathrm{y}, \mathrm{z})$ system should be established.

Numerous 3D touch systems which can be mainly divided into three categories have been presented so far. The first one is machine-based system where users should wear additional devices, as shown in Figure 1 [1]. Although 3D information can be obtained accurately, the machine-based system is thought to be inconvenient due to additional devices and large volume.

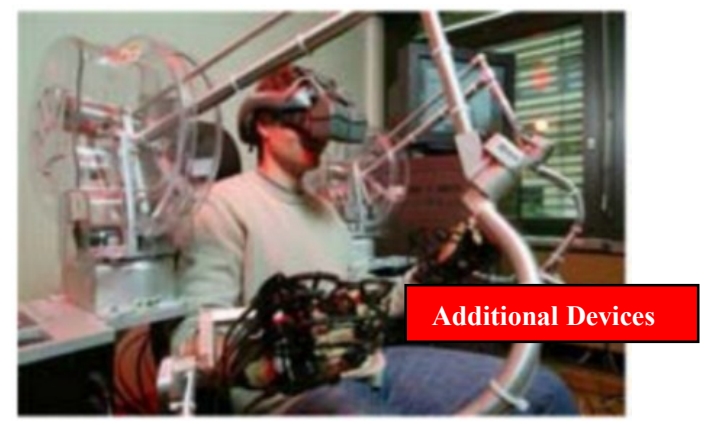

Figure 1. Machine-based system

Therefore, camera-based system in which users do not have to hold any inconvenient devices was proposed. In camera-based system, 3D information can be recognized due to the high resolution and autofocus of the cameras. However, the narrow viewing angle of cameras results in the blind range in near distance, which would not allow users to interact with portable display as shown in Figure 2a. Although TouchLight [2] was proposed, where cameras were set behind the display, to achieve near distance interaction; the system is still not suitable for portable devices due to large volume, as shown in Figure $2 \mathrm{~b}$.

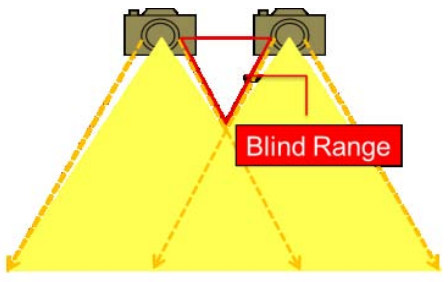

(a) Blind range issue

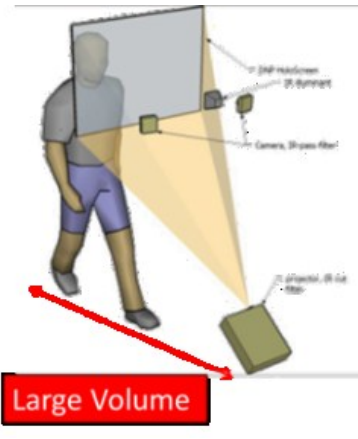

(b) Large volume issue
Figure 2. Camera-based system

\section{Motivation}

As discussed in previous section, both machine-based and camera-based systems are inappropriate for portable devices; consequently, optical-sensor-based system was proposed. Because the optical sensors could be embedded in the panel [3], not only thin form factor but also near distance interaction could be achieved. However, depth value (z) could not be directly obtained because the sensitivity and resolution of optical sensor are low; hence additional optical design is needed. Optical-sensor-based systems can be primarily classified into two types: lighting mode (light pen touch) [4][5] and reflecting mode (bare finger touch) [6][7][8][9]. In a light pen touch system, shown in Figure 3a, 3D information $(\mathrm{x}, \mathrm{y}, \mathrm{z})$ or even orientation $(\theta, \phi)$ could be retrieved due to the designed patterns on the light pen. However, light pen system is thought to be inconvenient owing to hand-held devices. Therefore, bare finger touch system providing a more intuitive and comfortable interface to users is one of the promising candidates for portable devices.

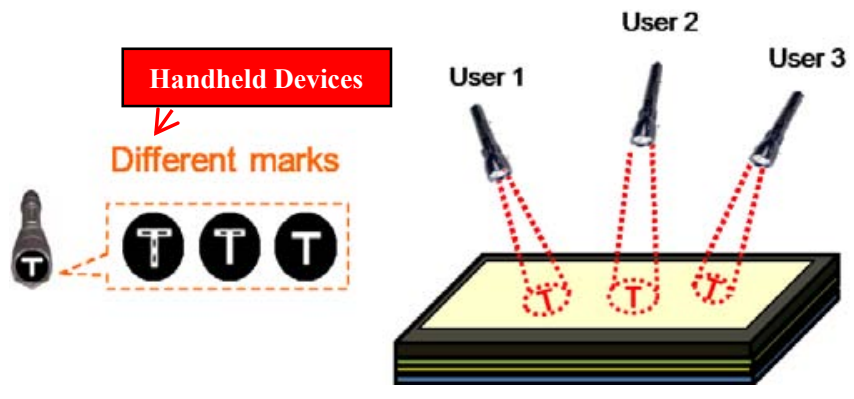

(a) Light pen touch system 


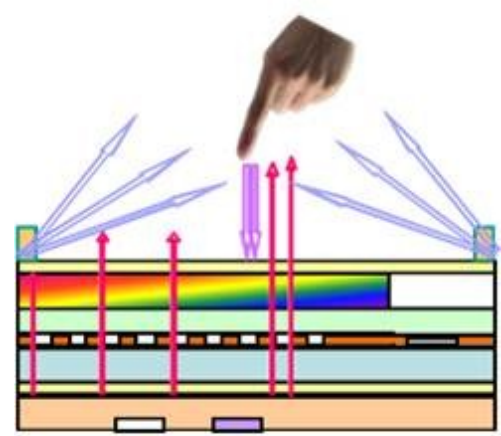

(b) Bare finger touch system

Figure 3. Optical-sensor-based system

A 3D finger touch with sequential illuminators [9] was proposed, and its construction is illustrated in Figure 3b. In the system, sequential illumination was carried out to detect 3D information; light was collimated at different tilt angles sequentially and the optical sensor captured reflected light synchronously. However, at least six frames were requested in order to get $3 \mathrm{D}$ coordinates, thus slow response became an issue. Slow response is an undesired feature of touchscreens; accordingly, our objective was to reduce the capturing time of the system by using parallel processing concept, multi-wavelength sensing. By utilizing parallel processing method, the information could be captured simultaneously instead of sequentially processed; the response time could be shortened, as shown in Figure 4.

\section{Original}
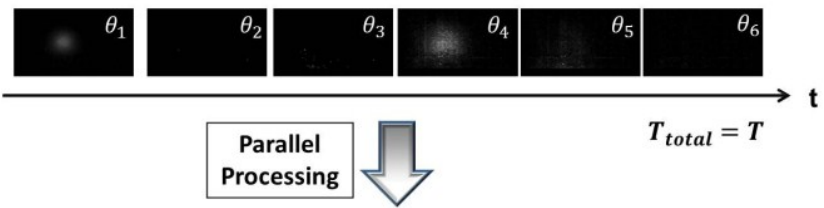

$T_{\text {total }}=T$

Processing

\section{Novel}

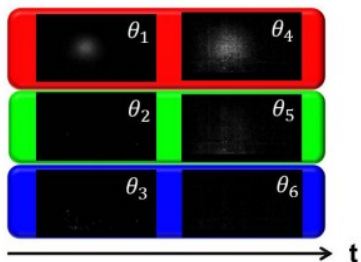

$T_{\text {total }}=\frac{1}{3} T$

Figure 4. Concept of reducing capturing time

\section{Multi-wavelength Sensing system}

In the multi-wavelength sensing system, different pieces of information of different tilt angles are captured simultaneously. In order to achieve multi-wavelength sensing, the construction of optical sensor is illustrated in Figure 5. Based on traditional display, the optical sensors are embedded on the TFT substrate; moreover, they are positioned under the color filter. Therefore, the optical sensors could sense red, green and blue light separately; hence the multi-wavelength sensors are introduced.

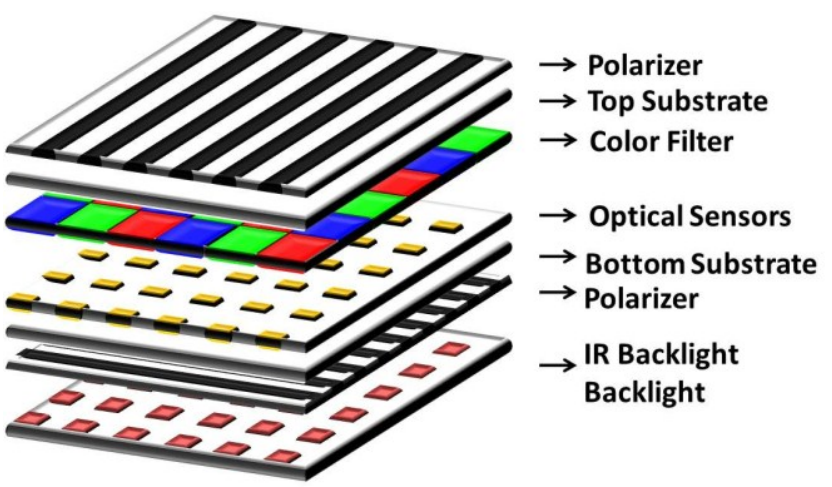

Figure 5. Touch panel with multi-wavelength sensors

To locate 3D position of an input object, multi-wavelength illumination is introduced, as shown in Figure 6. IR backlight, embedded in backlight system of display, is utilized to locate 2D coordinates $(\mathrm{x}, \mathrm{y})$; the side illuminators, constituting highly-collimated RGB LEDs line lights, are exploited to scan information of different tilt angles $\theta$. Moreover, at each frame, RGB line lights are emitted at different tilt angles simultaneously; and then the reflected light of different tilt angle could be captured by RGB optical sensors at the same time. Therefore, the capturing rate of the multi-wavelength sensing system could be up to as three times as that of the single wavelength (IR) one.

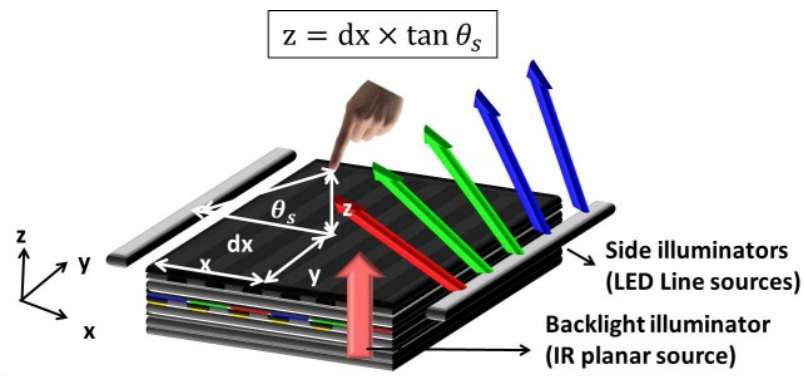

$\theta_{s}$ : Special tilt angle, the one having maximum overall intensity $\mathrm{d} x$ : Distance between illuminator and the object in $\mathrm{x}$-axis direction

Figure 6. Multi-wavelength illumination

Based on the panel with multi-wavelength sensors and multi-wavelength illuminators, the system agriculture is also shown in Figure 6. 2D coordinates $(\mathrm{x}, \mathrm{y})$ are defined as the projection of the finger onto the panel; $d x$ is the distance between side illuminator and the object along x-direction. $\theta_{\mathrm{s}}$ is special tilt angle at which light from side illuminator directly hit the object; and at the angle do the optical sensor receive an image with great intensity. In addition, depth value ( $\mathrm{z}$ ) is rectifiable through trigonometry.

\section{Algorithms}

Multi-wavelength sensing algorithm was proposed to analyze the images obtained from multi-wavelength illumination, as shown in Figure 7. In the beginning, images are classified into two groups, one is the image captured from backlight illumination, and the other is that form side illumination. All the images then are de-noised. After images being de-noised, overall intensity of the image from backlight illumination is used to judge whether the input object touched the panel or not. If the object touched the 


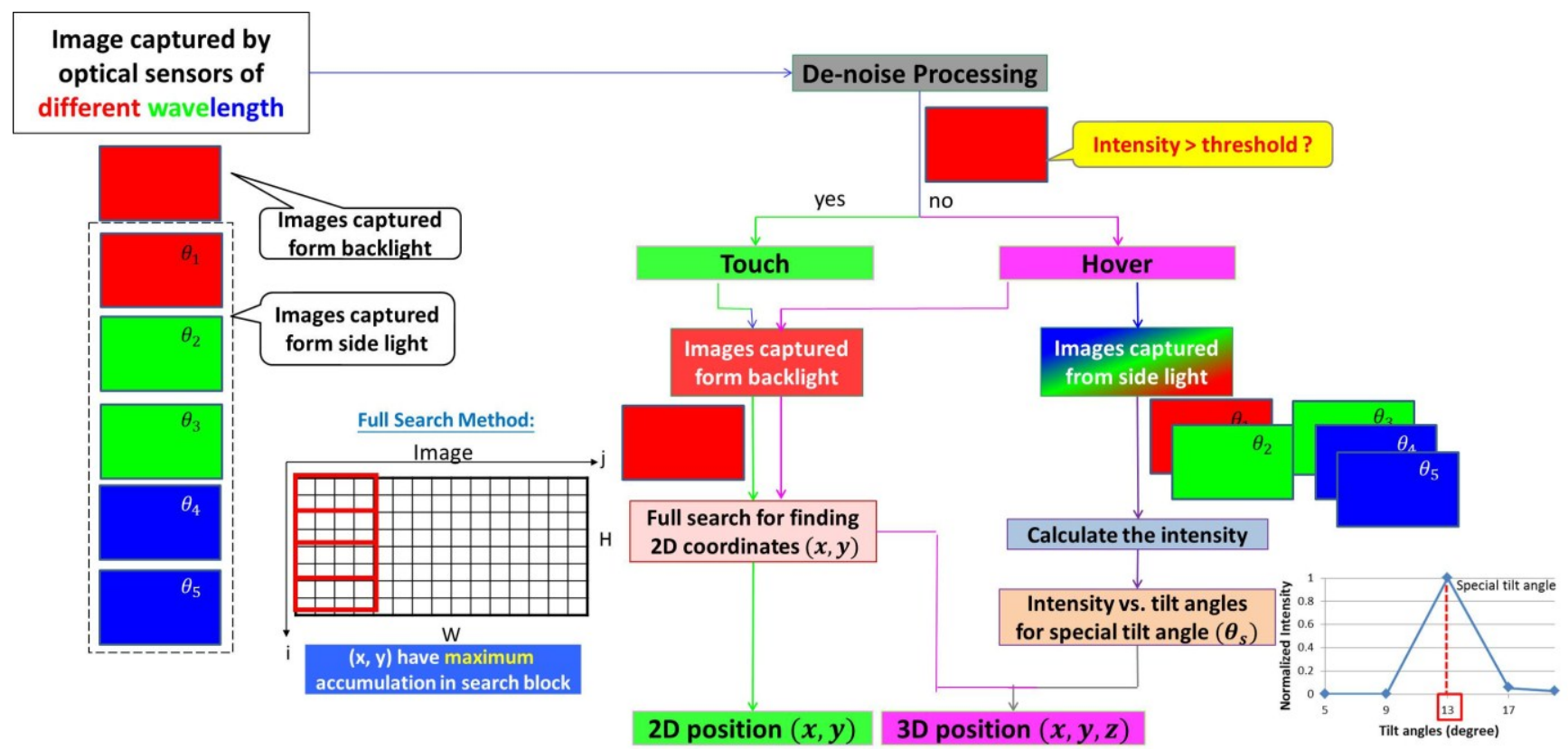

Figure 7. Flow chart of multi-wavelength sensing system

panel, full search is executed on the image, obtained through backlight illumination, to locate the $2 \mathrm{D}$ position $(\mathrm{x}, \mathrm{y})$ Otherwise, if the object hovered above the panel, full search is also executed to locate 2D coordinates; meanwhile, to search for depth value, the images from side illumination are individually calculated for their intensity. Besides, intensity of each image correlates to the tilt angle where it is captured. By comparing the intensity, the special tilt angle $\theta_{\mathrm{s}}$ is found to be the one with maximum intensity. Therefore, depth value $(\mathrm{z})$ can be calculated. Finally, $3 \mathrm{D}$ information $(\mathrm{x}, \mathrm{y}, \mathrm{z})$ is retrieved.

\section{Experiment Constructions}

Our proposed system was implemented on a 4-inch panel, where the optical sensors were embedded under red and blue color filter; pico-projectors were used as side illuminators, as shown in Figure 8a. And the software platform extracts signal from panel to form images for analysis, as shown in Figure 8b. In addition, because there were solely two sensors, red and blue lights were emitted in sequence, as illustrated in Figure 8c. At each frame, red and blue lights were collimated at different tilt angles simultaneously, and two images were captured by optical sensors separately. In addition, the working range of the system was set to be from 0 $\mathrm{mm}$ to $30 \mathrm{~mm}$ along $\mathrm{z}$-axis above the whole plane, which is feasible on portable devices.

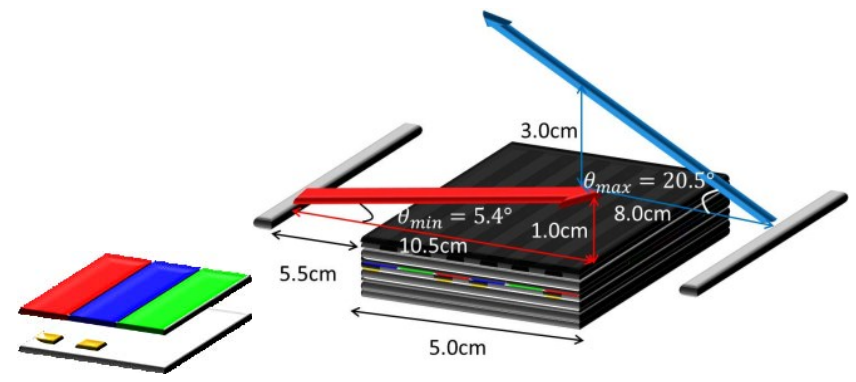

(a) Hardware platform

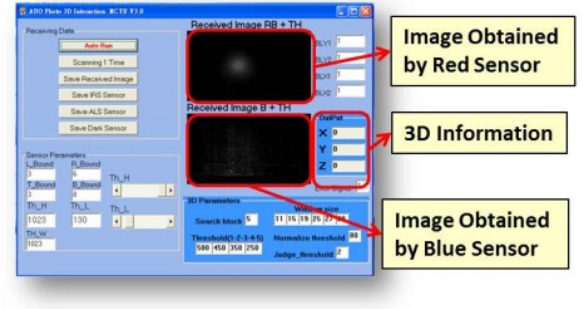

(b) Software platform

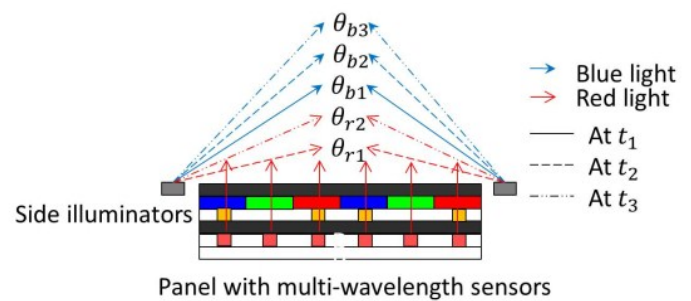

(c) Illumination sequence

Figure 8. Experimental platform

\section{Experiment Results}

\subsection{Z-axis Depth Accuracy}

To determine the resolution in xy-plane and z-axis, a scatter-reflected object, simulated as bare finger, was moved vertically along the $\mathrm{z}$-axis from 0 to $30 \mathrm{~mm}$ in $2.5 \mathrm{~mm}$ steps. For xy-resolution, images captured from backlight illumination are shown in Figure 9. The samples showed the maximum error was 2 $\mathrm{mm}$. On the other hand, for z-resolution, the response of calculated depth value vs. real depth value is shown in Figure 10. The maximum error was $4 \mathrm{~mm}$. Therefore, in reasonable working range of portable devices, the system could detect 3D information $(x, y, z)$ accurately. 


\begin{tabular}{|c|c|c|c|}
\hline $\begin{array}{c}\text { Real }(x, y) \\
(\mathrm{mm})\end{array}$ & $\begin{array}{l}\text { Scanned Image } \\
\rightarrow y\end{array}$ & $\begin{array}{l}\text { Calculated } \\
\quad(\mathrm{mm})\end{array}$ & $\begin{array}{l}\text { Error } \\
(\mathrm{mm})\end{array}$ \\
\hline $\begin{array}{r}\downarrow \\
(10,10)^{\mathrm{x}}\end{array}$ & 8 & $(8,10)$ & $(-2,0)$ \\
\hline$(10,78)$ & & $(9,78)$ & $(-1,0)$ \\
\hline$(25,45)$ & 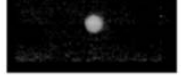 & $\stackrel{\bullet}{(24,76)}$ & $(-1,1)$ \\
\hline$(50,10)$ & & $(48,8)$ & $(-2,-2)$ \\
\hline$(50,78)$ & & ه & $(-2,0)$ \\
\hline
\end{tabular}

Figure 9. xy-resolution

\section{Z-resolution}

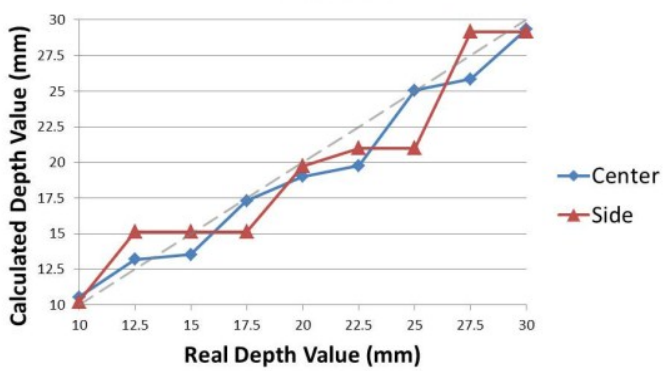

Figure 10. z-resolution

\subsection{Faster Gesture Response}

Finally, to certify that capturing time was successfully shortened, the original system and proposed system with multi-wavelength sensing were tested for their response time, as illustrated in Figure 11. In an ideal system, the sampling rate could be infinite, and the gesture could be fully reconstructed. However, in the original system where information was captured sequentially, the response was slow; hence the gesture recognized by original system was far from the real one. On the other hand, in the proposed system with red and blue sensors, capturing rate was as two times as the original one; hence the recognized gesture was much similar to the real one. Accordingly, the results indicated that real-time interaction in reflecting mode could be achieved through multi-wavelength sensing.

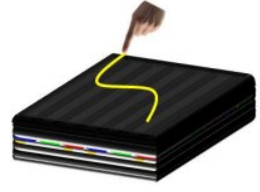

Hand gesture

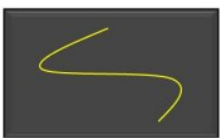

Real gesture

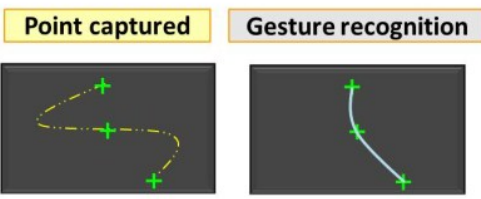

(a) Original system with single wavelength
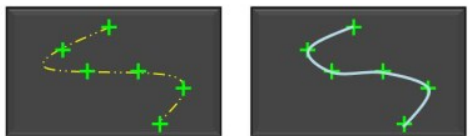

(b) Proposed system with multi-wavelength
Figure 11. Response comparison

\section{Conclusions}

We presented a bare finger 3-dimentional touch system with multi-wavelength sensing. The main contribution of this system was to reduce the capturing time, because the multi-wavelength can be illuminated and captured simultaneously instead of sequentially processed. Therefore, the system provides not only $2 \mathrm{D}(\mathrm{x}, \mathrm{y})$ touch but also $3 \mathrm{D}(\mathrm{x}, \mathrm{y}, \mathrm{z})$ touch. The working range along $\mathrm{z}$-axis is from 0 to $30 \mathrm{~mm}$ above the whole panel; resolution in xy plane is $2 \mathrm{~mm}$ while z-resolution is $4 \mathrm{~mm}$, which could be applicable for portable displays. Finally, the concept had already been successfully implemented on a 4 -inch LCD panel, and the demo video is shown in [10].

\section{Acknowledgements}

Our thanks to the AU Optronics Corporation, Taiwan and Industrial Technology Research Institute (ITRI) of Taiwan, under contrast 100-B-07, for useful discussions in this work. We'd like to express our appreciation to National Science Council, in Taiwan for financial support under contrast Academic Projects No. NSC 99-2221-E-009-066-MY3.

\section{References}

[1] Ott, R., M. Gutiérrez, D. Thalmann, and F. Vexo. "Advanced Virtual Reality Technologies for Surveillance and Security Applications." ACM, pp. 163-170, 2006.

[2] A. D. Wilson, "TouchLight: an imaging touch screen and display for gesture-based interaction," in Proceedings of the 6th international conference on Multimodal interfaces, State College, PA, USA, pp. 69-76, 2004.

[3] T.W. Pai, G.Z. Wang, Y.P. Huang, and J.M. Hung, "3D Interaction Display with Embedded Optical Sensors," Optics and Photonics Taiwan, 2008.

[4] G.Z. Wang, M.C. Ma, S.Y. Tung et al., "50.4: A Virtual Touched 3D Interactive Display with Embedded Optical Sensor Array for 5-axis (x, y, z, theta, phi) Detection," SID Symposium Digest of Technical Papers, vol. 42, no. 1, pp. 737-740, 2011.

[5] H.Y. Tung, G.Z. Wang, Y.P. Huang, "P-195: Multi-user and Multi-touch System for 3D-interactive Display," SID Symposium Digest of Technical Papers, vol. 42, no. 1, pp. 1834-1837, 2011.

[6] K. Yi, C. Choi, S. Suh et al., "45.2: Distinguished Paper: Novel LCD Display with a Sensible Backlight," SID Symposium Digest of Technical Papers, vol. 41, no. 1, pp. 673-676, 2010.

[7] C. Brown, D. Montgomery, J.L. Castagner et al., "31.3: A System LCD with Integrated 3-Dimensional Input Device," SID Symposium Digest of Technical Papers, vol. 41, no. 1, pp. 453-456, 2010.

[8] Y.P. Huang, G.Z. Wang, "3D Interactive Panel with Parallax Barrier" 3DSA 2010.

[9] M.C. Ma, G.Z. Wang, and Y.P. Huang, "P-199: 3D Finger Touch with Sequential Illuminator," SID Symposium Digest of Technical Papers, vol. 42, no. 1, pp. 1848-1851, 2011

[10] http://www.youtube.com/watch?v=0ngBKf3iqxmM 